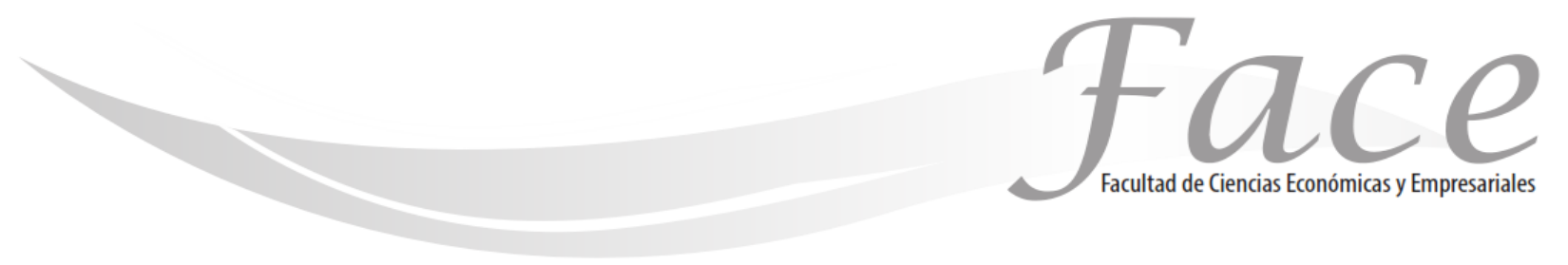

ISSN 1794-9920

Volumen $15-2$

Año 2015

Págs. 88 - 98

\title{
PROPUESTA DE MEJORAMIENTO EN LA GESTIÓN DE PEQUEÑOS PRODUCTORES CAPRINOS EN CAPITANEJO, SANTANDER-COLOMBIA *
}

Alix Antonia Macías Bermúdez **

Fecha de Recepción: 25 de Septiembre 2015

Fecha de Aprobación: 4 de Diciembre 2015

\begin{abstract}
Resumen:
Con el fin de proponer un plan de mejoramiento en la gestión de los pequeños productores caprinos en Capitanejo, Santander, Colombia, se realizó un estudio de caso exploratorio desde un enfoque descriptivo, por medio del cual se obtuvo información sobre aspectos socioeconómicos, de producción, comercialización y perspectivas de la gestión de la producción; encontrándose que esta es una actividad realizada artesanalmente, por tradición familiar y hace parte del medio cultural de la región del Cañón del Chicamocha, Colombia. Por su dinámica, las pequeñas producciones se identifican con el modelo de famiempresas, se desarrollan en una región con condiciones ambientales favorables para la caprinocultura, certificada como zona libre de aftosa y brucelosis, con un importante potencial para la producción. Con base en la metodología de planeación estratégica, se identificaron los factores internos y externos más relevantes y se identificaron factores estratégicos para el mejoramiento de la producción como la organización, la gestión del conocimiento y de la información; se considera que a través de la asociación de los pequeños productores caprinos y de la gestión de proyectos articuladores con organizaciones, entes territoriales, academia, etc., podrían lograrse importantes avances que contribuirían a mejorar los ingresos de las familias productoras y su calidad de vida.
\end{abstract}

Palabras Claves: producción caprina, famiempresa, gestión, planeación estratégica.

\footnotetext{
*Artículo resultado en el proyecto de Tesis de Maestría avalado por la Universidad los Libertadores de Bogotá. D.C

** Ingeniera Industrial, Especialista en docencia universitaria- USTA. Especialista en evaluación de la educación- UPTC. Ms (c). en Administración convenio UPTC-Universidad Nacional. , Sede Bogotá. D.C. Colombia. Contacto: aamaciasb@unal.edu.co
} 


\title{
MANAGEMENT IMPROVEMENT PROPOSAL FOR SMALL FARMS GOAT PRODUCERS IN CAPITANEJO, SANTANDER, COLOMBIA.
}

\begin{abstract}
:
In order to propose a management improvement plan for small farms goat producers in Capitanejo, Santander, Colombia, an exploratory case study was carried out using a descriptive approach, through which information on socioeconomic, production, commercialization, and management perspective in production was collected; and findings showed that it is a handicraft activity, a family tradition and is part of the cultural environment of the Chicamocha Canyon region, Colombia. Small scale producers identify themselves with the "family business" model, in a region with favorable environmental conditions for goat production, certified as FMD, brucellosis and tuberculosis free zone, with an important production potential. Based on the strategic planning methodology, the most relevant external and internal issues were identified as well as strategic factors for the organization's improvement such as production, knowledge and information management. It is considered that significant progress can be achieved through the association of small farms goat producers with companies, local authorities and academia, which will contribute to the improvement of the producers' families' income and their quality of life.
\end{abstract}

Keywords: Goat production, Family Business, Management, Strategic Planning.

\section{PROPOSTA DE MELHORIA DA GESTÃO DE PEQUENOS PRODUTORES DE CAPRINOS EM CAPITANEJO, SANTANDER-COLÔMBIA.}

\begin{abstract}
Resumo:
A fim de propor um plano de melhoria na gestão de pequenos produtores de caprinos em Capitanejo, Santander, Colômbia, um estudo de caso exploratório foi realizado a partir de uma abordagem descritiva, por meio do qual foram obtidas informações sobre aspectos socioeconômicos, produção, comercialização, e perspectivas da gestão da produção; sendo esta uma atividade artesanal, por tradição familiar e faz parte do ambiente cultural da região do Cânon do Chicamocha, Colômbia. Pela sua dinâmica, as pequenas produções se identificam com o modelo de empresas familiares, se desenvolvem em uma região com condições ambientais favoráveis para a caprinocultura, certificadas como uma zona livre de febre aftosa e de brucelose, com um importante potencial de produção. Com base na metodologia de planejamento estratégico, os fatores internos e externos mais relevantes foram identificados assim como os fatores estratégicos para a melhoria da produção como a organização, a gestão do conhecimento e da informação; considera-se que através da associação de pequenos produtores de caprinos e a gestão de projetos articulados com organizações, entidades territoriais e a academia, importantes avanços poderiam ser alcançados que contribuiriam para melhorar a renda das famílias produtoras e sua qualidade de vida.
\end{abstract}

Palavras-Chave: Produção Caprina, Empresa Familiar, Gestão, Planejamento estratégico. 


\section{INTRODUCCIÓN:}

La producción de caprinos en Colombia, ha sido tradicionalmente marginal y de naturaleza artesanal, con producción regionalizada (como en el Cañón del Chicamocha), donde la producción y el consumo son de carácter cultural. Con la escasa disponibilidad de tierra de algunos productores, de recursos de infraestructura y/o tecnología por parte de otros, la producción caprina se convierte en una alternativa de trabajo y consumo informal en algunas regiones del país (Castellanos et. al., 2010).

En el departamento de Santander, Colombia, especialmente en el Cañón del Chicamocha donde se encuentra Capitanejo, dice Salazar (2010), que este es un inmenso espacio de suelos semidesérticos, topografía muy quebrada, alta temperatura, humedad relativa muy baja, abundante material rocoso y vegetación propia de desierto con mucha espina, donde se producen excelentemente las cabras, especialmente la raza Santandereana, con gran sentido de reproducción y ejemplares totalmente adaptados a la zona.

Según el Instituto Colombiano Agropecuario (ICA) (2009), el país está certificado libre de aftosa y la provincia de García Rovira en Santander, a la que pertenece Capitanejo, está certificada desde julio de 2009 libre de brucelosis, condición sanitaria favorable para la producción caprina. Con la creación de la Cadena Productiva OvinoCaprina, CPOC, por parte del Ministerio de Agricultura y Desarrollo Rural, MADR, se proyectan importantes avances para la caprinocultura; en julio de 2012 la cadena suscribió el Acuerdo Nacional de Competitividad, en el cual se definen acciones y estrategias para el desarrollo competitivo y sostenible de este sector.

Con el fin de brindar un aporte a la gestión de la producción caprina en los pequeños productores del municipio de Capitanejo, se realizó un estudio de caso con enfoque cualitativo el cual tiene un alcance exploratorio y descriptivo, que pretende examinar un tema poco tratado cuyos propósitos son describir las tendencias de los sujetos de estudio en los aspectos socioeconómico, de producción, comercialización y de gestión, identificar los factores internos y externos más relevantes que inciden en ellos, y de su análisis establecer los aspectos estratégicos que pueden contribuir al mejoramiento de la gestión de estas pequeñas producciones, teniendo en cuenta las características favorables del entorno en que se encuentra, las particularidades de la carne y la leche apropiadas para el consumo humano y el mercado de productos caprinos. De igual manera, esta información puede convertirse en insumo para la toma de decisiones de los productores como también para la formulación de proyectos del sector.

\section{MARCO TEÓRICO:}

Importancia y función de la cabra en el mundo

La población de cabras está en gran parte en manos de pequeños productores (Espinal, Martínez y Amézquita, 2006); en los hogares rurales se encuentra el $90 \%$ de las cabras, estos hogares se caracterizan por la pobreza y débiles prácticas agrícolas. También carecen de habilidades de gestión modernos que son esenciales para mejorar la productividad (Morand-Fehr y Lebbie, 2004). Sin embargo, la cabra ha cumplido con las necesidades nutricionales de los países con incremento de población. Además, ha sido a menudo el punto focal de las costumbres religiosas y ha proporcionado oportunidades de empleo, incluso para los miembros más desfavorecidos de las sociedades pobres. El animal tiene una positiva imagen ecológica al igual que la calidad y el valor nutritivo de sus productos (Boyazoglu, Hatziminaoglou y MorandFehr, 2005).

Según Boza (2006), el $79 \%$ de la población caprina mundial se encuentra en áreas climáticamente clasificadas como desfavorecidas o áridas-cálidas, inadecuadas para otras actividades y en las que las producciones de caprinos son del mayor interés para numerosas explotaciones familiares. Por otro lado, las razas autóctonas están muy bien adaptadas a las condiciones locales, siendo capaces de soportar condiciones climáticas hostiles y prosperan con pastos de escasa calidad, con pequeños aportes de subproductos agrícolas, piensos y/o forrajes cultivados. Como se describe, es el caso de la condiciones en el Cañón del Chicamocha en particular.

Siguiendo a Morand-Fehr y Lebbie (2004), después de una industrialización exagerada de la agricultura, la cría de cabras está en condiciones de satisfacer las demandas sociales y las necesidades de los consumidores urbanos gracias a su buena imagen ecológica, a la calidad de la leche de cabra y productos lácteos bajos en grasa, más digerible y saludable, menos alergénica que la leche de vaca; también la calidad de la carne de cabra tiene menos grasa y colesterol que la de cordero u otras carnes rojas. La leche de estas especies es una valiosa fuente de proteína, grasa, calcio, hierro, fósforo, magnesio y vitaminas, particularmente la vitamina A (Boyazoglu et al., 2005).

En muchas zonas rurales en todos los continentes, las pequeñas explotaciones de subsistencia tienen pequeños rebaños con dos a diez cabras. Estas cabras son alimentadas con subproductos o pastan a lo largo de las carreteras 0 en los pastizales cercanos, muy a menudo los agricultores no tienen tierras dedicadas a la producción de 
forraje. Estas cabras, generalmente son cuidadas por mujeres y niños y son una fuente de ingresos complementarios a partir de la leche, la carne y las pieles. Estos sistemas no son generalmente parte de la economía de mercado, pero tienen un papel social importante para las poblaciones rurales pobres. La baja inversión necesaria para establecer estos sistemas es un punto a favor y una razón para su desarrollo. Ellos son probablemente uno de los sistemas responsables del reciente crecimiento del número de cabezas de ganado caprino en el mundo (Morand-Fehr et al., 2003, citado por Dubeuf, 2005).

Las cabras proporcionan leche, carne, fibras y piel, así como estiércol de alto valor como fertilizante (Iñiguez, 2004). A pesar del reconocimiento de las cualidades y el potencial de las cabras, esta especie parece ser menos favorable económica y comercialmente. Según Morand y Boyazoglu (1999), el sector caprino ha recibido significativamente menos apoyo que otros sectores de la producción animal, tales como el de la leche de vaca, carne de res, aves de corral o de cerdos.

De acuerdo con las estadísticas de la Organización de las Naciones Unidas para la Alimentación y la Agricultura (FAO/FAOSTAT) (FAOSTAT, 2009 citado por González et al, 2011), en el año 2007 se produjeron en el mundo cerca de 5 millones de toneladas de carne de cabra; sin embargo, su contribución a la oferta de proteína comercializable es muy baja, por el orden del 1\%; la comercialización de la carne de cabra se realiza localmente para el consumo doméstico y ventas informales, Dubeuf, Morand-Fehr y Rubino (2004). El consumo de carne y leche de cabra cumple un papel importante en la oferta de proteína en la dieta alimenticia de la población en zonas marginales. La producción de leche de los pequeños rumiantes representa el $3,5 \%$ de la producción mundial; esta participación es más importante en los países desarrollados donde el porcentaje de participación alcanza el $7,5 \%$, mientras que en los países en desarrollo es apenas del 1,5\% (Boyazoglu et al., 2005). Siguiendo a Dubeuf et al. (2004), en los países en desarrollo, un estudio de la situación muestra que probablemente menos del $5 \%$ de toda la leche comercializada se obtiene de las cabras, el sector de los productos lácteos de cabra es menos organizado o es una producción de doble propósito (carne y leche) y la mayor parte de la leche es consumida en el hogar o vendida localmente sin ninguna organización real.

\section{La producción de caprinos en Colombia}

De acuerdo con la Agenda de la Cadena Ovino Caprina del MADR, las ovejas y cabras tienen una importante función social para la población rural y comunidades indígenas del país, ya que proporcionan alimento, ofrecen medios para el mantenimiento cultural y de la economía rural, así mismo, tiene grandes posibilidades para suplir mercados altamente rentables. Sin embargo, no se cuenta con información pertinente de esta producción y se dispone de datos aislados (Castellanos et. al., 2010).

La FAO (2013), reporta a Colombia como el octavo país productor de carne ovina y caprina en América Latina con 15.000 toneladas. Según la Encuesta Nacional Agropecuaria (2011), en 2011 se estimó la población nacional caprina en 1.693 .531 animales, donde los departamentos de La Guajira, Cesar y Santander lideran la participación en el inventario caprino, aportando Santander el 29,4\%. Según el DANE (2011), en el periodo de 1991 2006, se exportaron 461 toneladas de productos caprinos, siendo el $97 \%$ carne caprina; estas exportaciones dirigidas hacia las Antillas Holandesas en más del $90 \%$ y en pequeña proporción a Estados Unidos, Perú, Uruguay y Venezuela (Espinal et al., 2006); en conclusión, el principal producto de exportación en el sector caprino es la carne. En la priorización de las Apuestas de la Agenda Interna de Competitividad y Productividad para Santander 2006 en la producción de proteína animal, los caprinos ocupan el tercer lugar, después de la producción avícola y la producción bovina (Departamento Nacional de Planeación 2007) y según el Sistema de Información de la Cadena Ovino-Caprina, SIOC, el inventario caprino en Santander en el mes de diciembre de 2014 fue de 53.962 cabezas, siendo en ese mes el segundo productor en el país después de La Guajira (MADR, SIOC).

La Dirección de Cadenas Productivas del MADR, indica que la Cadena Ovino Caprina tiene el reto de fortalecerse, especialmente estimulando las exportaciones e incentivando el mercado interno, de manera que su crecimiento sea significativo como el de ganado bovino. Con ese propósito, asociaciones de pequeños productores en Santander trabajan además de la venta de carne de cabra, en la transformación de la leche como una alternativa para la generación de ingresos (Sena, s.f.).

La cadena de ovinos y caprinos de Colombia es relativamente joven en comparación con cadenas de más trayectoria institucional. El gremio de productores se encuentra representando a nivel nacional desde el año 2000, por la Asociación Nacional de Caprinocultores y Ovinocultores de Colombia, ANCO, y su principal objetivo es el de propender por el desarrollo de esta producción en el país (Espinal et al, 2006).

Elementos de la gestión en las pequeñas producciones caprinas 
En las pequeñas producciones caprinas, las diferentes actividades involucradas en el proceso de producción son realizadas por los integrantes de la familia y su práctica es una alternativa de apoyo a su subsistencia. Según Segovia y Melgarejo (2011), las microempresas familiares son conocidas como famiempresas y se conciben como una acción de emprendimiento para reproducirse y sobrevivir en su propio ámbito rural, cuyo principal capital es la mano de obra de los integrantes de la familia, quienes desarrollan habilidades y destrezas adquiridas por transmisión de sus ascendentes; enfrentan las adversidades para mantenerse y buscan alternativas para mejorar su ingreso y calidad de vida. Se caracterizan por relaciones laborales basadas en el parentesco o acuerdos afectivos, poco o nulo nivel tecnológico, baja capacidad de gestión administrativa, organizativa y de producción. El emprendimiento empresarial se ha venido extendiendo en las economías en desarrollo, siendo de larga trayectoria el emprendimiento empresarial familiar rural.

Koontz y Weihrich (1998), abordan el proceso administrativo como el conjunto de actividades realizadas por personas que trabajando juntas buscan un fin específico y lo hacen de manera eficiente, lo cual aplica a todo tipo de organización y a todo nivel organizacional. La administración está basada en los valores de los empresarios y en el desarrollo del negocio que mejor conocen, en estructuras organizacionales simples con un mínimo de personal y sin dejar al azar el logro de los objetivos. Según Guerra y Aguilar (2002), la planificación estratégica es el proceso por el cual el gerente del agronegocio ordena sus objetivos y sus acciones en el tiempo, considerando que la estrategia es un aspecto fundamental en la gestión de una organización para la toma de decisiones, el uso óptimo de los recursos y el logro de las metas trazadas. "Mintzberg señala que el término estrategia ha sido definido de múltiples formas, pero casi siempre resulta común en ellas que es un conjunto consciente y deliberado de orientaciones las cuales determinan decisiones hacia el futuro" (p.237), (Montoya, 2009). Para Hax y Majluf (2004), la estrategia tiene un concepto multidimensional, con un sentido de unidad, orientación y propósito, en cual facilita las modificaciones necesarias inducidas por su medio.

Según Montoya, Montoya y Muñoz (2011), la aplicación del enfoque de metáforas biológicas en las organizaciones, es una metodología que permite mediante la asociación de términos de la sociobiología, obtener una mayor comprensión de los conceptos de formación comunitaria de la identidad territorial, entendida como la delimitación de un sistema de actividad humana, con propósito definido en un espacio denominado territorio, donde los individuos se afianzan con la apropiación de valores, sentimientos e instituciones que los distinguen como región y los convierten en distintivos de sus productos y servicios (Echeverri y Ribero, 1998 citados por Montoya et al., 2011).

\section{METODOLOGÍA:}

El estudio se abordó desde un enfoque cualitativo, a través del cual se aproxima a hacer una comprensión detallada de las perspectivas de otros dentro de su contexto (Taylor y Bogdan, 1984). Se ha seleccionado el estudio de caso exploratorio de tipo descriptivo, porque permite la búsqueda de información de la dinámica desarrollada por los pequeños productores caprinos en su propio contexto y facilita la comprensión y descripción del fenómeno. De acuerdo con Yin (2014), en este tipo de estudios se investiga un fenómeno contemporáneo en su contexto real y estos son ricos en descripciones empíricas de casos particulares que se basan en múltiples fuentes de información. Según Eisenhardt, 2007, los estudios de caso pueden albergar gran variedad de fuentes de datos, incluyendo entrevistas, datos de encuesta, observaciones, etc. Son recomendables cuando el fenómeno que queremos estudiar no puede ser comprendido de forma independiente respecto a su contexto, a su ambiente natural (Johnston, et al., 1999 citados por Villarreal y Landeta, 2010), es decir, cuando queremos comprender un fenómeno real considerando las variables que tienen relevancia en él (McCutcheon y Meredith, 1993 citados por Villarreal y Landeta, 2010). Según (Méndez, 2008), se complementan con la aplicación de herramientas como la entrevista, la observación directa, etc.

El presente trabajo tuvo como unidades de estudio nueve pequeñas producciones caprinas, siendo entrevistado el productor e integrantes de la familia partícipes en las diferentes actividades; se realizó en el municipio de Capitanejo, departamento de Santander, Colombia, en las veredas Quebrada de Vera, Montecillo y El Datal, consideradas en el Esquema de Ordenamiento Territorial entre las de mayor producción (Alcaldía Municipal de Capitanejo, Santander, 2003). Las unidades de análisis fueron seleccionadas mediante muestreo no probabilístico por conveniencia, el cual permite al investigador definir intencionalmente los sujetos de observación, donde el objetivo es la riqueza, profundidad y calidad de la información (Hernández, et. al., 2000); se tomaron tres pequeñas producciones por cada vereda (considerando pequeñas aquellas que tuvieran menos de diez animales), teniendo en cuenta las posibilidades de acceso a las mismas.

Para obtener la información se realizaron visitas de campo en las cuales se hizo observación directa de los aspectos 
tratados en las entrevistas semiestructuradas aplicadas a las personas que participan en las actividades en estas pequeñas unidades de producción seleccionadas, con el fin de obtener información pertinente para el análisis de las siguientes variables: socioeconómica (nivel educativo, tenencia de la tierra y fuentes de ingresos económicos); producción (razas, alimentación y manejo sanitario); comercialización (carne, leche, derivados y subproductos); perspectivas de los productores (para mejorar su pequeña unidad de producción).

\section{RESULTADOS:}

\section{Socioeconómicos}

En el manejo de las pequeñas unidades de producción participan todos los integrantes de la familia la cual está conformada entre 5 y 6 miembros, siendo las mujeres las principales encargadas de los caprinos dado su fácil manejo por el tamaño y mansedumbre de los animales, a los niños les asignan labores de pastoreo. El $89 \%$ de los productores tiene nivel de escolaridad de primaria siendo en algunos casos incompleta y no han recibido capacitación sobre la caprinocultura, excepto el 22,2\% correspondiente a dos productores que pertenecen a la asociación. La propiedad de la tierra es de minifundio, el $78 \%$ posee menos de 1 hectárea, los demás viven en arrendamiento; la agricultura es su principal fuente de ingresos y la producción caprina es considerada una fuente alterna para atender emergencias económicas 0 acontecimientos familiares en los que se acostumbra a celebrar con el plato típico de cabrito. En conclusión, la importancia está centrada en la producción de carne, aunque actualmente se le viene dando significado económico a la leche, como lo dice el Presidente de Asocaprica (A. Macías, comunicación personal, 28 de febrero de 2015): "la caprinocultura en Capitanejo se ha dedicado a la producción de carne para abastecer el consumo local y tradicionalmente la leche es para alimento de los niños y los cabritos; sin embargo, en los últimos cinco años se ha incrementado el consumo de leche en la población y su precio actualmente duplica el precio de la leche de vaca en la comercialización local, convirtiéndose en una expectativa de mercado atractiva para la generación de ingresos".

\section{Producción}

Las razas predominantes son la Santandereana, propia de la región y los cruces de ésta con Alpina, Nubiana y Saanen; las pequeñas producciones caprinas no tienen macho reproductor y los cabritos son vendidos en edades entre seis y ocho meses. Los animales son pastoreados en las orillas de los cultivos y caminos; el forraje disponible está conformado por especies nativas comúnmente conocidas como oreganillo, mulato, espino de cabro, cuji y gallinero; solo los productores asociados han plantado algunas especies forrajeras. En el aspecto sanitario reconocen la presencia de enfermedades parasitarias, casos de ectima y encefalitis por los que han tenido pérdida de animales; las enfermedades son atendidas por los mismos cabreros según su experiencia.

\section{Comercialización}

La producción se vende localmente, siendo las épocas de mayor demanda la semana santa, las fiestas patronales y navideñas, llegando en ocasiones a no tener oferta; los productores no conocen el comportamiento del mercado caprino en otras localidades. La comercialización es de animales vivos y el $22,2 \%$ sacrifica los animales en sus casas para vender la carne a consumidores directos 0 a la asociación; en cuanto a la leche, el $22,2 \%$ la vende cruda también a consumidores directos o a la asociación, los demás productores consumen una parte y otra parte es para los cabritos. Los subproductos como las pieles, se comercializan con un único comprador el día domingo (día de mercado); la caprinaza producida es utilizada en la fertilización de suelos para la producción agrícola.

\section{Perspectivas de los productores}

Los productores que tienen la experiencia de comercializar la leche manifiestan la expectativa de incrementar su producción para mejorar sus ingresos, ya que su precio por litro duplica actualmente al de la leche de vaca; los productores asociados esperan beneficiarse de un proyecto para establecer un sistema de riego como también llegar a prepararse en la transformación de productos lácteos y cárnicos. Las expectativas de los productores están acordes a la práctica que vienen realizando, las posibilidades que visualizan están relacionadas a la pertenencia o no a la asociación de productores.

\section{DISCUSIÓN:}

Dadas las características socioeconómicas de las pequeñas producciones caprinas, se asimilan a las famiempresas y en su direccionamiento juega un importante papel la mujer como emprendedora; así como lo afirman Segovia y Melgarejo (2011), en esta acción de emprendimiento su principal capital es la capacidad de trabajo de sus miembros, lo cual fue confirmado en las visitas de campo. Para estas familias, la producción caprina es considerada un ahorro del que pueden disponer por la fácil comercialización de la carne, concepto que se ha mantenido arraigado por tradición y cultura en la región, el cual puede reconsiderarse y convertirse en un factor de 
capitalización y generación de ingreso económico con mayor importancia para la familia, dado su fácil manejo, las condiciones ambientales y sanitarias favorables para la producción y las expectativas favorables que tiene del mercado para la carne, la leche y subproductos; según Udo, et al. (2011), estudios de caso muestran que la producción de pequeños rumiantes es como el ahorro que proporciona seguridad y ayuda a acumular capital.

Según la Agenda Prospectiva de Investigación y Desarrollo Tecnológico (Castellanos et al., 2010), a excepción del sector comercial, la cadena ovino-caprina se caracteriza por realizar actividades artesanales e informales con bajo nivel de tecnología para el desarrollo de sus productos, ha sido tradicionalmente marginal donde la producción y el consumo son de carácter cultural. De acuerdo con estas consideraciones, los pequeños productores caprinos de Capitanejo adolecen de condiciones de infraestructura física y tecnológica, de asistencia institucional, se ven limitados por las condiciones de minifundio de la propiedad y su capacidad de gestión como productores individuales es muy baja. El sacrificio de animales en la práctica de traspatio realizada informalmente, no permite un manejo adecuado de la carne en condiciones de inocuidad y bioseguridad; preocupa a los productores los costos que el servicio de sacrificio pueda tener si existiera, de igual manera tampoco hay condiciones adecuadas de manejo de la leche, condición que desfavorece su comercialización. Por la información obtenida de dos encuestados que pertenecen a la asociación, reconocen los beneficios obtenidos de la organización en el logro de nuevos conocimientos para mejorar la producción y comercialización de los diferentes productos caprinos.

La principal línea de producción es la carne, la cual desde el pastoreo adquiere un exquisito sabor gracias a la alimentación de los animales con plantas como el oreganillo, saborizante natural, que en términos de los productores, los animales al comerlo se "adoban vivos", condición natural que ha dado reconocimiento a la carne de los animales del Cañón del Chicamocha. El desconocimiento en algunos casos y no aplicación en otros, de buenas prácticas de manejo, asociado a la creencia que la cabra por su rusticidad se defiende sola, genera pérdidas económicas que afectan significativamente al pequeño productor.

En la producción caprina de la región, la raza Santandereana es el patrimonio más importante, la cual provee la base genética de la raza autóctona, adaptada a las adversas condiciones del Cañón del Chicamocha; según Boza (2006), desde un plano conservacionista, la práctica controlada del pastoreo involucra aspectos de la preservación natural, mantenimiento de la biodiversidad, conservación del patrimonio genético y salvaguarda de la razas autóctonas.
Los productos caprinos tienen muchas bondades por su carne y leche saludables para el consumo humano (proteína de mayor digestibilidad, no son tan ricos en ácidos grasos saturados y colesterol como el cordero y la carne de res), Dubeuf et al. (2004), por la suavidad y docilidad de las pieles en la fabricación de calzado, bolsos, carteras, maletas, prendas de vestir, etc., valores agregados de la producción caprina que se deben promover en el mercado para incrementar su consumo.

Con la información obtenida de los productores se identificaron en las pequeñas producciones caprinas los aspectos internos y externos más relevantes que inciden en la gestión, relacionados en la tabla 1.

Tabla $\mathrm{N}^{\circ} 1$.

Aspectos internos y externos presentes en las pequeñas producciones caprinas de Capitanejo-Santander

\begin{tabular}{|c|c|}
\hline ASPECTOS INTERNOS & ASPECTOS EXTERNOS \\
\hline Fortalezas & Oportunidades \\
\hline $\begin{array}{l}\text { Condiciones ambientales } \\
\text { favorables para la producción } \\
\text { caprina en la región del Cañón del } \\
\text { Chicamocha. }\end{array}$ & $\begin{array}{l}\text { Certificación sanitaria de la región } \\
\text { libre de enfermedades zoonóticas } \\
\text { (aftosa, brucelosis y en proceso de } \\
\text { certificación de tuberculosis). }\end{array}$ \\
\hline Buena adaptación y producción de & $\begin{array}{l}\text { Crecimiento potencial de la } \\
\text { demanda de productos caprinos. }\end{array}$ \\
\hline $\begin{array}{l}\text { Políticas favorables del MADR en } \\
\text { la cadena ovino caprina. }\end{array}$ & $\begin{array}{l}\text { Precios estables y en potencial } \\
\text { crecimiento. }\end{array}$ \\
\hline Debilidades & Amenazas \\
\hline $\begin{array}{l}\text { Bajo reconocimiento de la } \\
\text { producción caprina como fuente } \\
\text { importante de ingreso económico. }\end{array}$ & $\begin{array}{l}\text { Falta de apoyo institucional en } \\
\text { asistencia técnica e infraestructura } \\
\text { para el sacrificio de ganado. }\end{array}$ \\
\hline $\begin{array}{l}\text { Incumplimiento de normas } \\
\text { sanitarias, de inocuidad y } \\
\text { bioseguridad en el manejo de la } \\
\text { carne y la leche y productos } \\
\text { transformados. }\end{array}$ & $\begin{array}{l}\text { Mercados con exigencias de } \\
\text { calidad. } \\
\text { Monopolio del mercado de pieles. }\end{array}$ \\
\hline $\begin{array}{l}\text { Bajo nivel de asociación y } \\
\text { conocimiento en temas de gestión. } \\
\text { Bajo nivel de conocimiento en } \\
\text { prácticas de manejo de la } \\
\text { producción animal. }\end{array}$ & $\begin{array}{l}\text { Escasa disponibilidad del recurso } \\
\text { hídrico. }\end{array}$ \\
\hline
\end{tabular}

A partir del análisis del modelo DOFA, se enuncian en la tabla 2 las estrategias propuestas para contribuir en la mejora de los pequeños productores caprinos de Capitanejo. 
Tabla $\mathrm{N}^{\circ} 2$.

Estrategias del análisis DOFA en pequeños productores caprinos de Capitanejo Santander

\begin{tabular}{|c|c|}
\hline $\begin{array}{l}\text { Incrementar la producción de } \\
\text { materias primas de caprinos } \\
\text { (carne y leche) para abastecer la } \\
\text { demanda y prepararse para la } \\
\text { transformación. } \\
\text { Gestionar el proceso de } \\
\text { certificación de origen, teniendo } \\
\text { en cuenta las ventajas de la } \\
\text { región de procedencia. }\end{array}$ & $\begin{array}{l}\text { Estrategia DO } \\
\text { Fortalecer la organización a través } \\
\text { de la asociación y el } \\
\text { emprendimiento en las } \\
\text { famiempresas caprinas. } \\
\text { Fomentar la cultura de los registros } \\
\text { y en general del manejo de la } \\
\text { información de la unidad productiva. } \\
\text { Estandarizar los procesos para la } \\
\text { comercialización y proyecto de } \\
\text { transformación de leche, carne y } \\
\text { pieles. }\end{array}$ \\
\hline Estrategia FA & Estrategia DA \\
\hline $\begin{array}{l}\text { Capacitar a los productores en } \\
\text { buenas prácticas de producción } \\
\text { animal y buenas prácticas de } \\
\text { manufactura, fomentando la } \\
\text { cultura de la calidad. }\end{array}$ & $\begin{array}{l}\text { Brindar asistencia técnica } \\
\text { agropecuaria a los productores y } \\
\text { dar apoyo a sus organizaciones en } \\
\text { la formulación de proyectos } \\
\text { productivos (riego, sistemas } \\
\text { agrosilvopastoriles, etc.). }\end{array}$ \\
\hline $\begin{array}{l}\text { Capacitar a los productores en } \\
\text { procesos de transformación de la } \\
\text { leche, la carne y procesamiento } \\
\text { de pieles cumpliendo con normas }\end{array}$ & $\begin{array}{l}\text { Brindar condiciones de } \\
\text { infraestructura apropiada para el } \\
\text { sacrificio de ganado. }\end{array}$ \\
\hline $\begin{array}{l}\text { ambientales y de calidad } \\
\text { aplicables. }\end{array}$ & $\begin{array}{l}\text { Implementar el mejoramiento } \\
\text { genético acorde con las condiciones } \\
\text { ambientales y de adaptación de las }\end{array}$ \\
\hline $\begin{array}{l}\text { Promover y publicitar la } \\
\text { producción primaria como } \\
\text { estrategia turística del Cañón del } \\
\text { Chicamocha y la comercialización } \\
\text { de productos. }\end{array}$ & $\begin{array}{l}\text { razas caprinas. } \\
\text { Realizar alianzas estratégicas con } \\
\text { restaurantes, supermercados, } \\
\text { tiendas, etc. y establecer puntos de } \\
\text { venta de productos caprinos. }\end{array}$ \\
\hline
\end{tabular}

Fuente: Matriz de estrategias resultado del estudio

A partir del análisis de los aspectos internos y externos del modelo DOFA, se identificaron tres factores estratégicos en los que se enmarca la propuesta de mejoramiento de la gestión para la pequeñas unidades de producción 0 famiempresas caprinas: el primero la asociación, como eje integrador de los productores para fortalecer a la organización comunitaria y a las pequeñas unidades productivas; según Solarte (2011), la asociatividad es la estrategia de fortalecimiento de las unidades productivas, de los productores individuales y del producción y comercialización de sus productos como para mejorar sus ingresos y así su nivel de vida. El segundo, la gestión del conocimiento, como el intangible más importante de la empresa; Arias, Portilla y Villa (2007), la definen como la estrategia que convierte los valores intelectuales en valor añadido e incremento de la productividad y la capacitación como el conjunto de actividades a través de las cuales se incrementa el conocimiento; y el tercero, la gestión de la información, entendida como la disponibilidad de datos para la toma de decisiones; según González et al. (2011), desarrollar alternativas no computarizadas para productores para los cuales el uso de las tecnologías de información y comunicación estén fuera de su alcance, como los esquemas de investigación, participación y acción, donde los productores más hábiles inducen cambios en los menos hábiles con relación a la importancia de la gestión de la información.

\section{CONCLUSIONES:}

Las pequeñas unidades de producción caprina se asimilan a las famiempresas rurales, los integrantes de la familia están vinculados directamente al proceso productivo, es una práctica tradicional que hace parte de la cultura regional del Cañón del Chicamocha y de su subsistencia; es un territorio reconocido como productor caprino en el país, que puede convertirse en un distintivo potencialmente diferenciador teniendo en cuenta su reconocimiento por la producción y consumo de carne de cabro, siendo atractivo turístico del municipio. Se reconoce el liderazgo y emprendimiento de la mujer, integrante importante que puede considerarse factor clave en la gestión de la producción, hacia quien debe dirigirse especialmente la capacitación en aspectos organizativos y de manejo de registros, por su mayor permanencia en el hogar y su capacidad para desarrollar procesos asociativos y de manejo de la trazabilidad de la producción.

Las características generales de las pequeñas producciones caprinas se enmarcan en familias rurales económicamente pobres, con bajos niveles educativos, bajo uso de tecnologías, la región se caracteriza por el minifundio, terrenos áridos secos, rusticidad de la producción caprina por lo cual los productores consideran que los animales requieren poca atención, su saber tradicional prevalece y es la práctica común, por lo cual no hay interés en capacitarse; la connotación es de una producción de baja importancia económica y social. La caprinocultura en Capitanejo está centrada en la producción de carne, aunque la leche actualmente duplica el valor de la leche de vaca, la mayor parte de su producción se consume en los hogares y es alimento para los cabritos, siendo este renglón de proyección económica significativa sin descuidar su aporte a la generación de alimento para la familia.

Con el fin de alcanzar resultados económicos y sociales favorables para las pequeñas unidades de producción caprina, es necesario fomentar el espíritu asociativo, de manera que se potencialicen las capacidades individuales de los productores y se generen colectivamente oportunidades para la consecución de recursos externos que sean invertidos en el mejoramiento y desarrollo de la producción; con beneficio de la existencia de la cadena ovino caprina, su agenda de competitividad, la certificación 
que tiene la región como zona libre de las enfermedades de aftosa, brucelosis y en proceso de ser libre de tuberculosis, la existencia de la cabra Santandereana actualmente en proceso de certificación como raza, las características físicas y químicas de la carne y la leche de cabra por su alta digestibilidad de su proteína, grasa y carbohidratos, las ventajas comparativas de la región por sus condiciones geográficas y ambientales aptas para la producción caprina y el reconocimiento que por estas condiciones tiene a nivel nacional, etc., con una gestión organizada es posible en el mediano plazo llevar a cabo procesos de investigación aplicada que permitan la gestión de nuevos conocimientos y desarrollo de nuevas tecnologías y modelos ambientalmente sostenibles en las pequeñas unidades caprinas que pueda ser replicados en otras localidades, la formulación y ejecución de proyectos productivos dirigidos a los caprinocultores asociados.

Canalizar sus esfuerzos hacia mejores niveles de competitividad, requiere elevar el nivel de conocimiento de los productores y la visión del negocio, para lo cual la aplicación de la planeación estratégica como herramienta de gestión puede orientar el proceso hacia la implementación de acciones articuladas con diferentes actores como los productores, las asociaciones, las entidades territoriales, entes nacionales, la academia, etc., a través de proyectos integradores que conduzcan a mejorar la calidad de vida de las familias y sea la empresa caprina una importante fuente de ingresos. La capacitación como soporte de la gestión del conocimiento será el intangible más valioso en el proceso de mejoramiento, el cual incidirá en un cambio en la percepción sobre la importancia económica de la producción que podrá convertirse en un factor motivacional para generar valor agregado y desarrollar otros eslabones de la cadena que pueden orientar la producción hacia nuevos mercados.

\section{REFERENCIAS:}

Alcaldía Municipal de Capitanejo, Santander (2003). Esquema de Ordenamiento Territorial. Recuperado de http://cdim.esap.edu.co/BancoMedios/Documento s\%20PDF/eot-capitanejo-santander-2003tomo\%20iii-etapa\%20diagn\%C3\%B3sticoecon $\%$ C3\%B3mica-socialinfraestructura $\% 2$ fofuncionalpol\%C3\%ADtico\%20administrativa(138\%20p\%C3\%A1g\%20-\%20499\%20kb).pdf

Arias, L., Portilla, L., \& Villa, C. (2007). Gestión del conocimiento: el triunfo de los intangibles. Scientia et Technica, 1(35), 351-355
Recuperado

de http://revistas.utp.edu.co/index.php/revistaciencia/ article/view/5433/2865

Boza, J. (2006). Papel del ganado caprino en zonas desfavorecidas. Real Academia de Ciencias Veterinarias de Andalucía Oriental. Ponencia presentada en las XXX Jornadas Científicas de la SEOC, Granada, Septiembre 2005. Recuperado de

http://www.produccionbovina.com/produccion_ca prinal produccion_caprina/59zonas_desfavorecidas.pdf

Boyazoglu, J., Hatziminaoglou, I., \& Morand-Fehr, P. (2005). The role of the goat in society: Past, present and perspectives for the future. Small Ruminant Research. 60 (1-2),13-23.

Castellanos, J., Rodriguez, J., Toro., \& Luengas, C. (2010). Agenda prospectiva de investigación y desarrollo tecnológico para la cadena productiva cárnica ovino- caprina en Colombia. Bogotá: Minsterio de agricultura y desarrollo rural. Recuperado de http://asoovinos.org/prueba/wpcontent/uploads/2015/01/agenda_ovino_caprina1. pdf

DANE (2011). Encuesta Nacional Agropecuaria. Recuperado http://www.dane.gov.co/files/investigaciones/agro pecuario/ena/doc_anexos_ena_2011.pdf

Departamento Nacional de Planeación (2007). Agenda interna para la productividad y la competitividad en Santander. Recuperado de www.mincit.gov.co/descargar. php?id=61205

Dubeuf, J., Morand-Fehr, P., \& Rubino, R. (2004). Situation, changes and future of goat industry around the world. Small Ruminant Res. 51(2): 165-173.

Dubeuf, J. (2005). Structural, market and organisational conditions for developing goat dairy production systems. Small Ruminant Res. 60(1-2): 67-74.

Eisenhardt, K. \& Graebender, M. (2007). Theory building from cases opportunities and challenges. Academy of Management Journal, 50 (1), 25-32.

Espinal, C., Martínez, H., \& Amézquita J. (2006). La cadena ovina y caprina en Colombia. Documento de Trabajo No 125. Bogotá: Ministerio de Agricultura y Desarrollo Rural, Observatorio Agrocadenas de Colombia. Recuperado de 
Hugo Alfonso Hernández Escolar

http://bibliotecadigital.agronet.gov.co/jspui/bitstrea m/11348/3914/1/20078611357_caracterizacion_o vinosycaprinos.pdf

FAO (2013). FAO Statistical Yearbook 2013. World food and agriculture. Recuperado de http://www.fao.org/docrep/018/i3107e/i3107e03.p df

González, C.A., Grajales, H.A., Manrique, C. \& Téllez, G. (2011). Gestión de la Información en los Sistemas de Producción Animal -una Mirada al Caso de la Ovino-Caprinocultura. Revista de la Facultad de Medicina Veterinaria y Zootecnia, 58(3). Recuperado de http://www.revistas.unal.edu.co/index.php/remeve z/article/view/28908/29577

Guerra, G. \& Aguilar, A. (2002). La planificación estratégica en el agronegocio. México: Editorial Limusa.

Hax, A. \& Majluf, N. (2004). Estrategias para el liderazgo competitivo. De la visión a los resultados. Buenos Aires: Ediciones Granica.

Hernández, R., Fernández, C., \& Baptist, P. (2000) Metodología de la investigación. Segunda edición. Editorial Mc Graw Hill

Instituto Colombiano Agropecuario (ICA) (2009). García Rovira Libre de Brucelosis. Recuperado de http://www.ica.gov.co/Noticias/Pecuaria/2009/Gar cia-Rovira,-primera-provincia-del-paisdeclarad.aspx

Iñiguez, L. (2004). Goats in resource-poor systems in the dry environments of West Asia, Central Asia and the Inter-Andean valleys. Small Ruminant Res. 51(2), 137-144.

Koontz, H., y Weihrich, H. (1998). Administración. Una perspectiva global, 11 edición. México: McGraw Hill Interamericana editores.

Méndez, C. (2008). Metodología. Guía para elaborar diseños de Investigación en ciencias económicas, contables y administrativas. 4ta edición. México: LIMUSA.

Ministerio de Agricultura y Desarrollo Rural. Sistema de Información para la Cadena Ovino Caprina (2014). Recuperado de http://sioc.minagricultura.gov.co/index.php/opcdocumentoscadena?ide $=14$
Montoya, I., Montoya, L., \& Muñoz, G. (2011). Aplicación metafórica de elementos de la sociobiología en la formación comunitaria de la identidad territorial. En: I. Montoya y L. (Eds). Montoya Metáforas Biológicas Aplicadas a las Organizaciones (237252). Bogotá: Editorial Universidad Nacional de Colombia

Montoya, L. \& Montoya, I. (2012). Metáforas Biológicas Aplicadas a las Organizaciones. Bogotá: Editorial Universidad Nacional de Colombia

Morand-Fehr, P. \& Lebbie, S.H.B. (2004). Proposals for improving the research efficiency in goats. Small Rumiant Research, 51 (2), 145-153.

Morand-Fehr, P., \& Boyazoglu, J. (1999). Present state and future outlook of the small ruminant sector. Small Ruminant Res. 34(3): 175-188.

Montoya, I (2009). La formación de la estrategia en Mintzberg y las posibilidades de su aportación para el futuro.

Salazar, P. (2010). La Cabra, Gran Alternativa de Producción. Recuperado de http://www.engormix.com/MAovinos/articulos/cabra-gran-alternativaproduccion-t3042/p0.htm

Segovia, A., \& Melgarejo, Z. (2011). La famiempresa, un sistema vivo y abierto de desarrollo socioeconómico. Revista de la Facultad de Ciencias Económicas y Administrativas. Universidad de Nariño,12(2), 181-199

Sena (s.f). SENA Santander trabaja en el fortalecimiento de la cadena de producción caprina. Periódico SENA. Recuperado de http://periodico.sena.edu.co/productividad/noticia. php?i=265

Solarte, G. (2011). Asociatividad, empresarización, pactos territoriales, Claves del desarrollo de los territorios rurales. Bogotá: Corporación Latinoamericana Misión Rural. Recuperado de http://www.misionrural.net/publicaciones/pactos/e mpresarizacion_pactos_pdf.pdf

Taylor, K. \& Bogdan, M. (1984). Introducción a los métodos cualitativos de investigación. Editorial Paidós. Recuperado de https://books.google.es/ books?id=EQanW4hLHQgC\&pg=PA12\&dq=inves tigaci\%C3\%B3n+cualitativa+Bogdan\&hl=es\&sa= X\&ved=0CCAQ6AEwAGoVChMlj_16aGZyQIVRmseCh2I 
ISSN: 1794-9920 Julio - Diciembre 2015

Volumen 15 Número 2 Año 2015 Págs. 88 - 98

$X Q M B \# v=$ onepage \&q=investigaci\% $\mathrm{C} 3 \% \mathrm{~B} 3 \mathrm{n} \% 20$ cualitativa\%20Bogdan\&f=false

Udo, H., et al. (2011). Impact of intensification of different types of livestock production in smallholder croplivestock systems. Livestock Science, 139, 22-29

Villarreal L., O., \& Landeta R., J. (2010). El estudio de casos como metodología de investigación científica en dirección y economía de la empresa. Una Aplicación a la Internacionalización. Investigaciones europeas de dirección y economía de la empresa, 16 (3) 31-52

Yin, R. (2014). Case Estudy Research: Design and Methods. 5 Edición. Sage Publications, inc. 